\title{
Le Associazioni delle donne e i diritti dell'infanzia in Italia (1861-1930)
}

Riepilogo: Lo scopo di questo articolo è quello di presentare la diffusione delle istituzioni educative per i bambini piccoli italiani e il modo in cui è significativamente legato alle associazioni delle donne e alle iniziative di pensatori e pensatori illuminati. L'articolo ricostruisce la storia del dinitto all'educazione per la prima infanzia in Italia nel periodo tra l'Unità d'Italia (1861) e i primi decenni del XX secolo. Rivela come queste iniziative hanno sottolineato la necessità di fornire istituzioni educative per giovani ragazze e bambini, non solo a fini di cura o igiene; ma spesso con importanti principi educativi come l'attenzione agli spazi, i rapporti con le famiglie e altri. L'articolo sottolinea anche come l'associazione delle donne abbia anche combattuto duramente per un'adeguata formazione professionale di insegnanti e insegnanti.

Parole chiave: Storia dellinfanzia. Diritti dei bambini. Educazione per la prima infanzia. Storia delle donne.

\section{As Associações das Mulheres e os direitos da infância na Itália (1861-1930)}

Resumo: O objetivo deste artigo é apresentar a difusão das instituições de educação para as crianças pequenas italianas e a forma como ela está ligada significativamente a associações de mulheres e as iniciativas de pensadoras e pensadores muito sensiveis a este tema. $\mathrm{O}$ artigo reconstrói a história dos direitos à educação para a primeira infância na Itália no período entre a Unificação da ltália (1861) e as primeiras décadas do século XX. Revela como estas iniciativas enfatizaram a necessidade de garantir instituições com fins educativos para meninas pequenas e meninos pequenos, não somente com fins de assistência ou de higiene; mas muitas vezes, com princípios educacionais importantes como a atenção para os espaços, a relação com as famílias, entre outros. $\mathrm{O}$ artigo aponta ainda como a associação de mulheres também lutou muito para formação profissional adequada de professoras e professores.

Palavras-chave: História da infância. Direitos das crianças. Educação Infantil. História das Mulheres.

\section{Women's Associations and Children's Rights in Italy (1861-1930)}

Abstract: The aim of this article is to present the diffusion of educational institutions for Italian young children and the way in which it is significantly linked to women's associations and the initiatives of illuminated thinkers. The article reconstructs the history of the right to education for early childhood in Italy in the period between the Unification of Italy (1861) and the first decades of the twentieth century. It reveals how these initiatives emphasized the need to provide educational institutions for young girls and young boys, not only for the purposes of care or hygiene; but often with important educational principles such as attention to spaces, relationships with families, and others. The article also points out how the women's association also fought hard for adequate professional training of teachers and teachers.

Keywords: History of childhood. Child rights. Early Childhood Education. History of women.

1 Dipartimento di Scienze Umane per la formazione. Università degli Studi di Milano-Bicocca. E-mail: gabriella.seveso@unimib.it 2 Doutora em Educação pela Universidade de São Paulo - USP. Departamento de Educação da Escola de Filosofia, Letras e Ciências Humanas, Universidade Federal de São Paulo - UNIFESP. E-mail: dfinco@unifesp.br 


\section{Donne, associazioni femminili e infanzia alla fine dell'Ottocento}

$\mathrm{I}$

n Italia il dibattito sulle istituzioni per la prima infanzia si sviluppa soprattutto a partire dalla metà dell'Ottocento attorno alla problematica delle condizioni dei bambini e delle bambine e delle loro madri: "Nel corso dell'Ottocento - scrive Carlo Corsini - si moltiplicano i dibattiti, i congressi, nazionali e internazionali, sulla protezione dell'infanzia, sull'igiene dell'allattamento, sull'assistenza e la beneficenza all'infanzia, sul lavoro minorile e sulla tutela della madri lavoratrici'” (Corsini, 1996, p. 264). Questi dibattiti scaturivano dalla constatazione delle condizioni sovente drammatiche in cui si trovavano i/le bambini/e e le donne delle classi lavoratrici e contadine, soprattutto a seguito del primo affermarsi di un'economia industriale. In alcuni casi, le riflessioni erano sollevate da politici e da pensatori che in realtà erano spinti dalla preoccupazione di mantenimento dell'ordine sociale o addirittura di controllo delle classi lavoratrici al fine di implementare il profitto imprenditoriale. In altri casi, però, le proposte nascevano dall'iniziativa filantropica e/o da manifestazioni caritatevoli che vedevano coinvolte e attive protagoniste le donne di classi sociali più agiate; in altri ancora, cominciava a farsi strada una rivendicazione mossa da maggiori consapevolezze politiche e sociali. Anche nel caso di iniziative filantropiche, comunque, si ottenne sovente il risultato di porre all'attenzione dell'opinione pubblica il tema importante della tutela dell'infanzia e del sostegno alle madri, e anche di realizzare prime forme di educazione infantile: queste ultime avevano il merito di trasmettere alle classi più disagiate elementi di cura che non avrebbero potuto conoscere in altro modo e di dare forma alle prime sperimentazioni che costituirono un esempio e un modello dal quale partirono poi realizzazioni più mature e più consapevoli.

In questa cornice, si distinsero le opere di alcune donne che appartenevano a classi sociali più elevate e che si proposero di diffondere i principi di cura e di attenzione all'infanzia e di sostegno alle madri lavoratrici. Nel 1850 a Milano, nel quartiere di Santa Cristina e due anni dopo in zona di Porta Ticinese, furono aperti due "ricoveri": il primo deve la sua fondazione all'opera instancabile e feconda di Laura Solera Mantegazza, affiancata dal filantropo Giovanni Sacchi: la donna, che apparteneva ad una famiglia alto borghese di Milano, decise infatti di fondare il Ricovero dei bambini lattanti, utilizzando e riadattando alcuni locali al piano terreno della sua casa con l'ingresso dalla Contrada Santa Cristina (poi rinominata Via Mantegazza). Laura si era già distinta per le sue azioni filantropiche, quando, a seguito delle Cinque Giornate, si era dedicata all'assistenza e alla cura dei feriti, riuscendo a raccogliere alcune somme per sovvenzionare le loro cure, grazie al suo elemosinare casa per casa. Dopo il 1850, la Solera Mantegazza decise di dedicarsi con impegno e con continuità ad opere umanitarie a favore delle donne, fondando la Scuola per adulte analfabete, La Scuola professionale femminile, e l'Associazione Generale di Mutuo Soccorso per le operaie milanesi. Resasi conto della necessità di offrire aiuto alle madri lavoratrici per la 
cura e l'allevamento dei figli, fondò il Ricovero di Contrada Santa Cristina per sostenere le madri sia dal punto di vista materiale, sia dal punto di vista del benessere dei/delle bambini/e. Il ricovero, già dopo un mese dalla fondazione, offriva ospitalità e cura a ben 40 bambini, le cui madri erano impegnate nell'industria della seta, negli opifici, nelle fabbriche di tabacchi e di fiammiferi, e come lavoranti nelle botteghe (Catarsi, 1982 p. 150). In questa istituzione, certamente erano presenti molti aspetti e finalità igieniche e sanitarie, ma era molto evidente anche l'attenzione al benessere psicofisico, alla cura degli spazi e dei materiali: erano messi a disposizione giochi e giocattoli e i/le bambini/e potevano fruire di un giardino ove muovere i primi passi con l'ausilio di sostegni. Questa istituzione, pur avendo alcuni limiti (per esempio, richiedeva alle madri dei lattanti di recarsi presso il ricovero alcune volte durante la giornata e questo non era facile per le lavoratrici degli opifici), era però sorretta dall'idea che la prima infanzia aveva diritto ad essere tutelata, a crescere all'interno di spazi adeguati e pensati, a poter fruire di cure e di attenzioni sia sul piano psicofisico sia sul piano educativo.

Nel corso dell'Ottocento, purtroppo, queste istituzioni, che sorsero ad opera di donne molto impegnate in una intensa attività sociale, furono anche oggetto di critiche accese: risulta interessante osservare come queste critiche rimasero le stesse per tutta la seconda metà del XIX secolo, ma anche nel Novecento, fino agli anni Settanta e oltre. Gli oppositori dell'educazione della prima infanzia, infatti, sottolineavano gli alti costi di queste istituzioni, e il timore che esse contribuissero a mettere in crisi la famiglia tradizionale; in particolare, sovente venivano rimproverate le donne lavoratrici, accusate di dedicarsi troppo agli impegni extradomestici.

Negli ultimi decenni del XIX secolo, inoltre, i nidi furono al centro di riflessioni molto controverse, relative alla loro funzione: all'interno di queste istituzioni, infatti, era presente sia personale femminile addetto alla cura dei/delle bambini/e, sia medici e sanitari che le rendevano strutturate anche con un taglio igienistico. La proposta di questi ultimi era di accorpare allinterno dei nidi, anche piccoli ospedali, oppure asili per accogliere madri nubili e loro figli/e o anche sale per ricoverare bambini abbandonati: queste proposte, purtroppo, ottenevano come risultato di rendere i primi nidi istituzioni poco percepite come educative e ricche di potenzialità positive per bambini/e e famiglie e più percepite come luoghi di assistenza per situazioni di difficoltà.

Nonostante le critiche e le non poche difficoltà, i primi nidi per l'infanzia si diffusero soprattutto nel Nord Italia, a Varese, Venezia, Trieste, Verona ed erano fondati con aspettative e con intenti educativi anche elevati, anche se purtroppo in quasi tutti i casi si trovavano poi a svolgere funzioni di custodia, a causa delle difficoltà finanziarie in cui versavano. Essi, infatti, vedevano la luce grazie all'opera di privati e private cittadini/e, che solo raramente ottenevano qualche finanziamento pubblico. In alcuni casi, invece, l'intervento illuminato di alcune donne permetteva la realizzazione di istituti per bambini e bambine della fascia di età 3-6: a seguito della diffusione delle teorie di Fröbel, tradotte in Italia da Adolfo Pick, ad esempio, a Venezia alcune donne appartenenti alla borghesia ebraica (Adele Della Vida, Laura Goretti Peruda, fra le altre) diedero vita ai primi giardini fröbeliani. Queste sperimentazioni, avviate da personalità 
illuminate e attente agli stimoli culturali provenienti dall'Europa, ebbero il merito di sollevare un dibattito interessante sia relativamente alle metodologie utilizzate per l'educazione infantile, sia relativamente ai diritti dell'infanzia: asili e asili nido in questi casi erano promossi da donne che sottolineavano gli aspetti educativi e non di mera custodia o assistenza, e che intendevano i loro interventi come rispondenti al diritto all'educazione di bambini e bambine e non solo come opere di beneficenza e di carità. Come sottolinea Tiziana Pironi in una densa e significativa ricostruzione, la diffusione del metodo fröbeliano in Italia fece sì che molte donne si impegnassero o nella realizzazione di iniziative a favore dell'educazione infantile o nei dibattiti vivaci che si svilupparono intorno ai metodi adottati nelle istituzioni per bambini/e. L'autrice ricorda a questo proposito l'opera del periodico "La Donna", fondato da Gualberta Alaide Beccari a Padova nel 1868: "Il giornale si distinse nella campagna a favore dei Giardini fröbeliani, in vista del superamento della concezione custodialistica dell'asilo infantile, sottolineandone invece l'esplicita valenza educativa" (Pironi, 2014, p. 26).

Le donne o le associazioni di donne che si impegnarono in questo cammino furono più di una $\mathrm{e}$ si diffusero anche in Italia centrale. A questo proposito, possiamo ricordare a titolo di esempio l'opera di Ernesta Galletti Stoppa nella zona di Ravenna: questa appassionata mazziniana si dedicò con fervore per tutta la vita a iniziative in favore delle donne, per promuovere l'istruzione femminile, la partecipazione politica, la consapevolezza e l'autonomia delle lavoratrici. Si impegnò inizialmente nell'associazionismo femminile, nella convinzione che solo la promozione sociale delle donne avrebbe permesso loro di trovare soluzioni concrete ai bisogni materiali e morali di una società che stava affrontando la diffusione dell'economia industriale e le sanguinose lotte per l'indipendenza. Resasi conto della tragica situazione in cui versava l'educazione di bambini e bambine in Italia in quel periodo, la Galletti Stoppa decise di fondare un istituto comprensivo, articolato in un giardino di infanzia, una scuola elementare e un corso di perfezionamento femminile: le opposizioni e le chiusure che incontrò da parte di alcuni ambienti conservatori la costrinsero a realizzare questo progetto a proprie spese. (Pironi, 2014). La novità e originalità di questa iniziativa consisteva nella realizzazione di un percorso formativo per bambini/e dai due anni e mezzo (giardino di infanzia) fino alla scuola elementare, aperto a tutti e non solo ai bisognosi: quest'ultimo aspetto dimostra come la fondatrice intendesse l'educazione di bambini e bambine non come opera di assistenza alle classi disagiate, ma come un diritto di tutti/e. La Galletti Stoppa riuscì a realizzare un giardino molto attento agli spazi, aereati, salubri, lontani dal traffico cittadino, ove bambini e bambine potessero dedicarsi anche ad attività manuali e di giardinaggio.

Questa ed altre iniziative mostrano come le donne o le associazioni femminili cercassero con vigore sia di mettere in atto vere e proprie sperimentazioni, sia di avviare riflessioni e dibattiti che favorissero il passaggio da un'ottica assistenzialistica e custodialistica delle istituzioni per la prima infanzia ad un'ottica educativa e centrata sui diritti.

Purtroppo, lo Stato italiano, che versava in condizioni economiche difficili e che si trovava ad affrontare problemi sociali molto complessi, non dimostrava invece di accogliere queste istanze e di 
considerare con sensibilità e attenzione la questione del diritto educativo di bambini e bambine della fascia prescolare. Sottolineiamo, infatti, come la Legge Casati (promulgata nello Stati piemontese, ma estesa a tutta l'Italia all'indomani dell'Unità), non considerava gli asili di infanzia e li definiva "istituti di carità": essi erano dunque di competenza del Ministero degli Interni e questo dato risulta assai significativo nel mostrare la chiusura degli ambienti politici nei confronti dei diritti educativi dell'infanzia.

Contemporaneamente, continuavano a diffondersi alla fine dell'Ottocento anche istituzioni rivolte a bambini e bambine della fascia di età 3-6: prime fra tutti, gli asili aportiani, che ebbero buona diffusione nel corso del secolo soprattutto in Italia del Nord, pur essendo avversati da alcuni importanti esponenti politici o della cultura del tempo (un curioso esempio è costituito da Giacomo Leopardi che scrisse un pamphlet proprio riguardo alle opere di assistenza e beneficenza all'infanzia).

Questi fermenti e queste iniziative erano peraltro sicuramente influenzate anche dalle riflessioni culturali e pedagogiche e dai fenomeni che si stavano sviluppando in quel periodo in altri Paesi europei. Ricordiamo che in Francia si erano già diffuse le écoles maternelles, con la funzione di offrire uno spazio educativo ai/alle più piccoli/e, sostituite nel 1881 da una legge che istituiva le salles d'asyle, con intenti quasi più vicini all'impostazione scolastica che a quella educativa. In Europa, inoltre, nel corso dell'Ottocento, si era sviluppato un dibattito intorno al tema della maternità e al suo valore sociale, al ruolo pedagogico ed educativo della donna: temi, questi, che si intrecciavano con quelli del diritto all'educazione di bambini e bambine. Certamente ebbe una sua influenza su questi temi anche la diffusione del pensiero di Pestalozzi, che riconosceva alla madre un ruolo prioritario e per la prima volta attribuiva all'opera educativa della madre una capacità di riflessione: l'amore materno, negli scritti pestalozziani, era "amore pensoso", ovvero sentimento non innato né connesso al semplice istino, ma capacità di riconoscere il proprio agire, di trasformare in sapere le tensioni affettive. Questa forte rivalutazione del sentimento materno riconosceva per la prima volta alle donne un sapere seppure non formalizzato, attribuiva loro compiti importanti di rigenerazione della famiglia e della società. Queste riflessioni contribuivano in maniera molto significativa ad una decisa valorizzazione della centralità educativa dei primi anni di vita di bambini e bambine e comportavano anche il concetto estremamente importante di supporto alla genitorialità.

I concetti che fanno da filo conduttore all'interno delle opere pestalozziane e in particolare in Leonardo e Gertrude, Come Gertrude istruisce i suoi figli, Lettera sullinfanticidio, sono straordinariamente vicini a quanto possiamo ritrovare negli scritti di alcune donne appartenenti ai movimenti e alle associazioni della fine dell'Ottocento e dell'inizio del Novecento, le quali ribadiscono con vigore l'importanza sociale del ruolo materno. Anche la diffusione del metodo fröbeliano ad opera di alcune appassionate estimatrici, finì per saldare il tema dei diritti educativi di bambini e bambine e dei metodi didattici più adeguati con quello del ruolo sociale della donna. Molte donne, inoltre, cominciarono ad interessarsi alle tematiche dell'educazione della prima infanzia a seguito della diffusione del fröbelismo: Tiziana Pironi ricorda come le divulgatrici e le sostenitrici di questo metodo erano in molti casi donne di classi sociali elevate, colte, a volte di origini non italiane, che si appassionavano all'idea di un rinnovamento educativo (Pironi, 2014). 
Le istanze educative infatti esprimevano

"un'esigenza che si coniugava con la maturazione di una coscienza etico-civile, - scrive Tiziana Pironi - che trovava espressione nell'idea, di derivazione mazziniana, di madrecittadina, ovvero di donna volta a interpretare la funzione materna come impegno sociale ed educativo nei confronti non solo dei propri figli, ma dell'intera società" (Pironi, 2014 p. 26)

L'inizio del Novecento e il diffondersi delle iniziative a favore dei diritti educativi dell'infanzia

Il dibattito politico si fece più vivace all'inizio del Novecento e prese in considerazione le istituzioni e le iniziative per la prima infanzia con posizioni alterne e non sempre chiare; alla fine del XIX secolo, lo Stato italiano tentò di regolamentare la situazione : una legge del 1890 mutava il vocabolario giuridico, abolendo la dizione "opere Pie" e introduceva la dizione di "Istituzioni pubbliche di beneficenza": si voleva in tal modo svincolare l'assistenza dalla carità, sottolineando una diversità di valori e la necessità di un approccio più razionale e il fatto che non era più possibile affrontare il problema dei bisognosi in termini di pietà. La filantropia fu sostituita, quindi, gradatamente, nei primi anni del XX secolo, da un più deciso intervento dello Stato e dai primi tentativi di una politica sociale che prendesse in considerazione i problemi dell'infanzia da un lato, la tutela della maternità dall'altro (Seveso, 2003, p. 132).

$E^{\prime}$ in questo clima che all'inizio del Novecento, si diffuse ulteriormente con vigore l'associazionismo femminile, che si occupò con sensibilità e con attenzione della condizione di miseria delle donne, dell'infanzia abbandonata, dei diritti all'educazione di bambini e bambine; in questo periodo, inoltre, forti personalità femminili riuscirono a ricoprire importanti cariche all'interno di Istituzioni di beneficenza, e a dare impulso ad iniziative assai significative. ${ }^{3}$ Tale associazionismo permise alle donne di impegnarsi in alcuni ambiti della vita sociale, superando le remore e gli impliciti divieti di ambienti conservatori: l'associazionismo femminile propose un'immagine di donna molto impegnata, attiva, informata ed istruita, capace di soccorrere ma anche di dibattere sulle problematiche sociali e di tentare di proporre soluzioni. É da sottolineare, inoltre, come l'associazionismo femminile si mobilitò proprio in ambiti all'interno dei quali le principali vittime di disagi sociali erano le donne, maggiormente sfruttate nell'ambito lavorativo, con un carico di lavoro e responsabilità enorme in ambiti familiare, e maggiormente esposte a rischi sanitari o a rischi sociali.

La storia delle istituzioni per la prima infanzia, quindi, si intrecciò in maniera indissolubile a quella dei movimenti delle donne, che si dimostrarono molto ferventi e determinate nel richiedere il riconoscimento del diritto alla educazione e alla cura dei/delle più piccoli/e. A questo proposito, occorre sottolineare come, accanto alle iniziative che sorsero in questo modo, in Italia videro la luce iniziative parallele, ad opera di industriali che si ispiravano ai modelli messi in atto negli opifici inglesi:

"All'alba del nostro secolo, in alcune industrie femminili, per favorire una più sana crescita dell'infanzia - scrivono Franco Cambi e Simonetta Ulivieri - si allestiscono

${ }^{3}$ Molte donne, inoltre, si impegnarono all'interno di Associazioni sorte non da iniziative femminili: all'interno della Società Umanitaria, per esempio, fondata nel 1912 grazie al lascito di 12 milioni di lire del magnate Prospero Moisé Loira, con scopi di beneficenza e di diffusione della cultura, militavano molte donne che si dimostrarono assai attive nel partecipare alle iniziative. 
delle camere di allattamento, dove a orari prefissati, qualche familiare (quasi sempre una figlia più grandicella) reca il piccolo; in altre, molto all'avanguardia, vengono aperte delle crèches, degli asili dove i bambini sono seguiti da donne o da religiose a spese della ditta e dove le lavoratrici possono allattare i propri figli durante i riposi concessi. Queste istituzioni sono determinate e motivate da un filantropismo imprenditoriale, che spesso affonda le sue radici nella necessità previdente di avere in futuro lavoratori sani da poter impiegare, e non un'umanità piena di patologie e incapace di produrre per debilitazione fisica" (Cambi, Ulivieri, 1988, p. 212).

In questo modo sorsero a Milano le strutture dell'industria cartiera Binda e quelle dell'industria di porcellane Richard, a Pistoia quelle di Cini, a Schio di Rossi. Queste iniziative, però, che erano a volte di livello buono, non partivano dalla rivendicazione dei diritti dell'infanzia e delle donne lavoratrici, ma piuttosto dalla convinzione degli imprenditori che fosse più produttiva una madre serena e meno impegnata con i figli o anche dalla necessità che le donne sposate con figli non uscissero dal mondo della produzione industriale, allinterno del quale erano utili per l'esperienza accumulata. Si tratta di una notevole e significativa differenza che caratterizza le opere di industriali rispetto alle iniziative realizzate dalle donne e dalle associazioni femminili: queste ultime si muovevano infatti nella ferma convinzione che andasse perseguito l'obbiettivo del diritto all'educazione dei/delle bambini/e ed erano ben distanti dalle motivazioni imprenditoriali degli industriali pur definiti “illuminati".

La situazione delle sale di allattamento fu ratificata con la legge 242 del 1902. Essa può essere considerata la prima legge a tutela del lavoro delle donne e dei fanciulli, costituita da una sorta di mediazione fra la proposta socialista e quella governativa: essa, rivelando notevoli ritardi e arretratezze rispetto ad altri paesi europei, elevava a dodici anni l'età minima per accedere al lavoro industriale e a quindici quella per esercitare il lavoro notturno; vietava, inoltre, alle donne e ai minori il lavoro nelle miniere e limitava l'orario giornaliero a dodici ore per le donne e a undici per i minori; prevedeva, infine, un congedo mensile per parto e una cassa per la maternità; non viene menzionato l'assegno per congedo di maternità. Nonostante l'approvazione rappresentasse comunque un passo avanti, in realtà, l'applicazione della legge era non facile e alcune richieste proposte dalle associazioni femminili, prima fra tutte l’Unione Femminile Italiana di Ersilia Bronzina Majno, non furono prese in considerazione.

A proposito delle sale di custodia previste e attuate all'interno delle fabbriche, inoltre, $\mathrm{i}$ movimenti delle donne intervennero sovente con consapevolezza e con conseguente atteggiamento critico, sottolineando come esse non avevano alcuna intenzionalità pedagogica, ma si preoccupavano solamente di mettere le donne nella condizione di essere più produttive all'interno della fabbrica stessa. Le donne stesse, dunque, giunsero a sottolineare che c'era una notevole differenza fra queste iniziative e quelle proposte e messe in atto da donne e/o da gruppi femminili, che invece erano sorretti dalla consapevolezza dei diritti all'educazione della prima infanzia e della necessità di tutelare e di supportare la maternità. In questa direzione si mosse anche una celebre attivista quale Anna Kuliscioff, che nei suoi discorsi più volte ribadì come la maternità non fosse percepita nel suo valore sociale, ma purtroppo solo come evento privato della donna, e come le madri fossero abbandonate a se stesse nell'affrontare il parto e la cura dei/delle 
figli/e $e^{4}$ Nella direzione di una rivendicazione dei diritti dell'infanzia si mosse anche l'Unione Nazionale Femminile, che si propose come scopi principali la creazione di circoli, ricreatori, biblioteche, e la formazione di persone che si dedicassero alle opere di assistenza e di previdenza, la diffusione dell'istruzione femminile, il miglioramento della condizione giuridica ed economica della donna; la difesa dell'infanzia della maternità, la raccolta di notizie e dati statistici. Nel marzo 1905, l'associazione assunse inquadramento giuridico di cooperativa e si diede per statuto obiettivi di assistenza e promozione umana e sociale. Il programma dell'Associazione metteva in luce anche l'obiettivo di unificare gli sforzi di diversi movimenti per l'emancipazione femminile, senza preclusioni legate all'appartenenza politica e/o sociale:

“All'intento di riunire le buone volontà e concentrare e coordinare le buone opere è sorta l'idea di fondare la Casa dell'Unione Femminile. Questa dovrà diventare la sede delle associazioni femminili, senza distinzione dei loro conformi o difformi caratteri politici o religiosi, istituzioni che si propongono di aiutare la donna per metterla materialmente e intellettualmente in grado di compiere la sua alta missione d'amore, di rigenerazione sociale".

A testimonianza dell'attenzione verso l'infanzia, occorre ricordare che nel 1908, partecipando ad un Congresso Nazionale sui temi dei minori, Ersilia Bronzini Majno, fra le fondatrici dell'Unione, propose una vivace e interessante relazione introduttiva sui diritti dell'infanzia, ribadendo la necessità di stabilire a cosa avessero diritto i/le fanciulli/e per uscire da una concezione tradizionale fondata sula carità e sulla pietà. A questo proposito, la Bronzini Majno, sia riguardo ai diritti dei bambini/e, sia riguardo ai diritti delle donne, si batté costantemente ribadendo la necessità di una gestione razionale degli enti di assistenza e delle iniziative, l'esigenza ineludibile di una accurata ed adeguata formazione del personale, l'urgenza di uscire da atteggiamenti moralistici e di considerare invece il tema dei diritti sotto l'aspetto sociale.

L'associazionismo femminile e le iniziative delle donne a favore dei diritti di bambini e bambine furono certamente influenzati dagli scritti di Ellen Key, divulgati in Italia all'inizio del Novecento da Sibillla Aleramo: la scrittrice svedese aveva infatti proposto riflessioni molto articolate e profonde riguardo alla condizione femminile e al "materno" e aveva saldato il tema del ruolo della donna con quello dell'educazione infantile. Questa autrice sosteneva l'importanza della ricerca di un'autenticità e di una reciprocità nella relazione fra uomini e donne e la necessità di una emancipazione femminile che non fosse una semplice copiatura del ruolo maschile; attribuiva, inoltre, al materno un forte valore culturale, intendendolo come forza presente nelle donne che può portare alla rigenerazione della società: "il materno si qualificava perciò come quella "differenza" con cui le donne avrebbero messo il loro sigillo su tutto il modo di pensare, di sentire, di volere e di agire" (Pironi 2010, p. 49). La Key poneva l'accento sulla dimensione etica della riflessione sul ruolo delle donne e sulla dimensione dei diritti dei bambini e delle bambine, ribandendo la necessità di uscire da un'ottica di carità e di assistenzialismo. Queste argomentazioni ebbero un'eco molto consistente in Italia e trovarono le donne e le associazioni femminili

${ }^{4}$ Anna Kuliscioff, laureatasi in Medicina dopo aver studiato nelle università svizzere e a Napoli e specializzatasi in ginecologia presso le università di Torino e Padova, provò, grazie alle ricerche per la sua tesi, l'origine batterica delle febbri puerperali, giungendo ad una scoperta che salverà in seguito migliaia di donne dalla morte per parto. Istituì, quindi, a Milano, in Via San Pietro all'Orto, un ambulatorio ginecologico gratuito, e si dedicò alle visite a domicilio o in loco delle pazienti più povere, nonché alla diffusione delle più elementari norme di igiene e di profilassi, venendo presto soprannominata la "dottora dei poveri". 
italiane molto pronte ad accoglierle. Possiamo trovare, ad esempio, alcune riflessioni molto simili in uno scritto di Placida Stefanini del 1913:

\begin{abstract}
"si sa che sulle ginocchia della madre che si formano i caratteri dei fanciulli, dalla madre si ricevono i primi impulsi i quali hanno talvolta un'influenza preponderante su tutta la vita. I figli diventeranno ottimisti o pessimisti a seconda dell'educazione ricevuta dalla madre. E la donna che mette i germi dei futuri sentimenti nei cuori dei figli: se questi germi non sono buoni, nulla di morale, di sano svilupperanno. Educhiamo la donna e collocheremo una scuola in ogni famiglia..." (Stefanini, 1913).
\end{abstract}

Il dibattito sull'educazione per la prima infanzia conobbe poi, all'inizio del Novecento, una formidabile e instancabile voce nell'opera di Maria Montessori. A questo proposito, vorremmo ricordare come la biografia di questa autrice dimostra lo stretto legame fra riflessione sui diritti dell'infanzia, attenzione ai diritti delle donne, impegno attivo per l'emancipazione femminile. La Montessori, infatti, partecipò intensamente alle iniziative dei movimenti di donne dell'inizio del Novecento: nel 1896 prese parte al Congresso Internazionale delle donne che si tenne a Berlino sottoponendo all'attenzione il tema dell'analfabetismo infantile e della disparità economica delle lavoratrici; nel 1899 si recò a quello di Londra come rappresentante delle donne italiane; al Convegno nazionale di Padova del 1899 propose una relazione focalizzata sul concetto di "femminismo scientifico" e molto determinata nel sostenere l'importanza che le donne fossero coinvolte nel cammino dell'istruzione e raggiungessero alti gradi di istruzione. L'autrice ricordava, infatti, come la preparazione culturale della donna non interferisce con il suo impegno familiare, ma anzi le permette di essere una madre più consapevole. Anche al Congresso di Roma del 1908 la Montessori ribadì la necessità di sostenere il lavoro extradomestico femminile per garantire alle donne l'autonomia e l'indipendenza nelle scelte di vita, in particolare per poter impegnarsi in una relazione matrimoniale che non fosse basata sul calcolo, La celebre pedagogista, ponendosi in stretto rapporto con altre importanti voci dei movimenti italiani (Bronzini Majno, Kuliscioff ecc.) e anche con esponenti straniere (prima fra tutte, Elle Key, con la quale ebbe un interessante epistolario), si impegnò con estrema dedizione per la causa del diritto delle donne al voto, partecipando alle principali iniziative del 1906. L'attenzione all'educazione dei bambini e delle bambine, infatti, correva parallela nei suoi scritti e nelle sue iniziative, alla notevole sensibilità per il tema del ruolo delle donne nella famiglia e nella società.

Non è possibile in questa sede analizzare specificamente e in maniera esaustiva e articolata il pensiero di Montessori, ma sottolineiamo alcuni aspetti significativi ai fini della presente trattazione: in particolare, vorremmo ricordare come anche nel caso di questa celebre autrice, la sensibilità e l'attenzione ai diritti dell'infanzia si saldarono al tema dell'emancipazione delle donne. La Montessori sottolineò inizialmente la correlazione fra appartenenza sociale e risultati scolastici, rilevandola nelle sue prime ricerche e mettendo in luce come bambini e bambine fossero quindi comunque persone inserite in un mondo sociale che forniva o meno loro occasioni adeguate e stimolanti di crescita e come tutti/e avessero però diritto all'educazione.

L'attenzione alla situazione sociale delle famiglie e la conseguente sottolineatura del ruolo scientifico ed etico dei servizi educativi e della scuola, restò molto presente nel pensiero montessoriano: 
non a caso, la prima casa dei bambini sorse a Roma in un quartiere disagiato nella zona periferica di San Lorenzo, ove le famiglie vivevano sovente in condizioni di miseria e di degrado.

La Casa di Bambini propose in maniera rivoluzionaria e radicale l'idea che i bambini e le bambine hanno diritto ad un ambiente educativo, salubre, non di mera custodia: la concezione sottesa è quella di bambino/a come piccolo/a scienziato/a, che esplora l'ambiente circostante, formula ipotesi e ha diritto a uno spazio a sua misura. Non meno importante è l'accento posto sulla bellezza e sull'estetica: bambini e bambine hanno diritto a uno spazio bello, curato armonioso; infine, la Montessori ribadiva come bambini e bambine fossero i/le futuri/e cittadini/e e quindi avessero diritto a sviluppare la loro autonomia e il loro senso critico fin da subito.

Il tema della emancipazione femminile era giocato in maniera molto significativa nelle opere montessoriane:

\begin{abstract}
"alla "donna nuova" - scrive a questo proposito Manuela Gallerani - spetta la duplice missione di socializzare su di un versante le virtù domestiche e diffonderle nella società, insieme ai valori di amore e pace che tradizionalmente sono costruiti in famiglia; sull'altro ha l'onere di concretarli e reintepretarli attraverso lo specifico contributo femminile" (Gallerani, 2010 p. 101).
\end{abstract}

Queste riflessioni ponevano la Montessori in profonda sintonia con altre esponenti della cultura femminile del tempo e con altre associazioni femminili: "l'esperimento montessoriano, con la socializzazione della funzione materna, realizzava di fatto quel concetto di maternità sociale avanzato dal femminismo del tempo, fornendo una risposta concreta alle perplessità sul lavoro extradomestico delle madri avanzate da Ellen Key" (Pironi, 2010, 69-70).

La considerazione dell'importanza cruciale del ruolo materno di esplicitava, nella realizzazione montessoriana, anche nell'attribuzione di un ruolo notevole all'interno della Casa dei Bambini: "la socializzazione della funzione materna puntava al diretto coinvolgimento delle madri, non solo con colloqui ed incontri settimanali, ma invitandole a visitare la Casa dei bambini in qualsiasi momento della giornata (Pironi, 2010, p. 68).

\title{
Le richieste relative alla preparazione delle maestre/educatrici
}

Le iniziative dei movimenti delle donne dell'inizio del Novecento, oltre che rivolte ad offrire istituzioni educative per la prima infanzia, si concentrarono sul problema della preparazione professionale del personale che prestava servizio nei nidi e nelle scuole dell'infanzia: molto spesso, infatti, si trattava di personale volontario, oppure assunto da coloro che avevano dato vita allistituzione, ma senza alcuna regolamentazione chiara a livello nazionale, in alcuni casi, la preparazione era per lo più sanitaria, in altri si trattava di una preparazione fondata essenzialmente sulla buona volontà e sul desiderio di prestare assistenza.

Le richieste avanzate dai movimenti delle donne erano sovente molto attuali: la riqualificazione dei percorsi formativi per le educatrici, la possibilità di aggiornamento, l'innalzamento degli stipendi; per quanto concerne le istituzioni educative. Per quanto concerne invece gli asili, le donne sottolineavano che 
avrebbero dovuto essere resi laici e gratuiti, a carico dei Comuni, dotati di materiali didattici, locali igienici, spazi verdi, gestiti da personale qualificato. In particolare, alcune associazioni o alcune esponenti di movimenti femministi chiedevano che fosse riconosciuta la valenza educativa dell'asilo anche ai fini di definire la preparazione del personale e che tali istituzioni fossero sottratte ad un'ottica assistenzialistica, precorrendo rivendicazioni degli anni Sessanta e Settanta: l'asilo avrebbe dovuto essere riconosciuto non come luogo di ricovero per bambini/e abbandonati da madri povere e lavoratrici, ma come vero e proprio segmento del sistema scolastico e le educatrici come vere e proprie professioniste con competenze educative e non meramente sanitarie. Ricordiamo, a questo proposito, l'opera significativa di Linda Malnati, insegnante e direttrice, scrittrice, sindacalista, consigliera di amministrazione in istituti ed Opere Pie, che denunciava con vigore la scarsità degli stipendi delle educatrici di asilo, l'arretratezza delle strutture, l'ottica assistenzialistica con cui queste istituzioni venivano gestite:

"Quale rimedio? - si chiedeva in un articolo del 1912 la Malnati a proposito dei nidi milanesi - ecco il problema da porre allo studio e da risolvere presto: la municipalizzazione degli asili infantili. È questa l'invocata riforma già in uso in alcune città e in alcuni borghi d'Italia. Solo con questa soluzione sarà garantita ai bimbi del lavoratore la prima educazione, data come diritto, non concessa come un'elemosina. E le donne del popolo, chiedono, per mezzo nostro, la difesa delle proprie creature, affidate ora ad Asili che trascinano una vita stentata, con personale mal retribuito, in locali inadatti nei quali è pressoché impossibile una educazione razionale, e igienicamente sana” (Malnati, 1912, p. 2).

Come è evidente in queste righe, la Malnati sottolineava l'esigenza di vedere l'educazione della prima infanzia come un diritto dei bambini e delle bambine e non come una concessione pietosa o come un atto di generosità: per questo motivo, si rivelava necessario rendere gli asili ambienti salubri, curati, ordinati, sereni, in cui operassero educatrici preparate, dotate di competenze pedagogiche, costantemente aggiornate.

Il tema della preparazione delle maestre fu affrontato più volte e con vigore anche da Maria Montessori, sia nei sui scritti, sia nei suoi interventi. Ai Congressi di Londra e di Berlino, questa autrice denunciò con determinazione la situazione di sfruttamento e di disagio nella quale si trovavano molte maestre in Italia: esse infatti si trovavano ad esercitare la professione in paesi sperduti, abbandonati, con un magro stipendio, spesso nell’isolamento sociale e senza possibilità di formazione (Seveso, 2001). La Montessori ribadì la necessità di formare educatrici che coniugassero rigore scientifico, consapevolezza, attenzione alla relazione e che avessero diritto ad una costante possibilità di aggiornamento. Le sue opere mostrano costantemente l'importanza di questa dimensione:

"la maestra - scrive l'Autrice - tuttavia ha molte e non facili mansioni: la sua cooperazione è tutt'altro che esclusa: ma diventa prudente, delicata e multiforme. Non abbisognano le sue parole, la sua energia, la sua severità, ma quel che occorre è la sapienza occulta nell'osservare, nel servire, nell'accorrere o nel ritirarsi, nel parlare o nel tacere, secondo i casi e i bisogni. Essa deve acquistare una agilità morale, che finora non le fu chiesta da nessun altro metodo, fatta di calma, di pazienza, di carità e di umiltà" (Montessori, 1980, p. 165)

In questo ritratto, possiamo osservare come la Montessori sottolinei la necessità di un cambiamento radicale nella preparazione professionale delle educatrici e delle maestre, preparazione che fino 
a quel momento era o sbilanciata sul versante igienico (per le scuole dell'infanzia) o molto connessa con i principali rudimenti delle discipline (per le scuole elementari). La maestra ed educatrice montessoriana è invece formata all'osservazione, alla riflessione sul proprio ruolo, alla capacità di entrare in relazione e porsi in ascolto. É da sottolineare come nelle opere montessoriane emerga però con chiarezza che le doti e le competenze delle educatrici non devono essere appiattite solo su una vaga capacità di relazione, ma devono essere affinate e sviluppate come continua autoriflessione sulle proprie azioni, a tal punto che l'autrice giunge a scrivere che ogni scuola si trasforma in un "gabinetto scientifico" (Montessori, 1986, p. 111) e ricorda come la maestra si possa definire una sorta di fusione fra il santo e lo scienziato:

"la veggenza della maestra dovrebbe essere insieme esatta come quella dello scienziato e spirituale come quella del santo. La preparazione alla scienza e la preparazione alla santità, dovrebbero insieme plasmare un'anima nuova, perché l'attitudine della maestra deve essere insieme positiva, scientifica e spirituale" (Ead. Ibidem, 121)

Non rientra fra gli scopi di questo contributi un'analisi approfondita di questi temi nell'opera montessoriana, ma vorremmo sottolineare come queste istanze presenti nella Montessori fanno eco e si collocano sula scia delle rivendicazioni interessanti e molteplici che le donne e le associazioni femminili portarono avanti nei primi due decenni del Novecento.

\section{La fondazione dell' O.N.M.I.}

A fronte di queste iniziative che si diffusero soprattutto alla fine dell'Ottocento e all'inizio del Novecento, l'avvento del Fascismo in Italia comportò una apparente attenzione alle rivendicazioni proposte dai movimenti delle donne e una politica che invece vanificò le conquiste che erano state raggiunte. Nel 1925, il regime affrontò il problema dei diritti dell'infanzia e della tutela della maternità fondando l'Opera Nazionale Maternità Infanzia (O.N.M..I.), un Ente parastatale che inizialmente era di beneficenza e di assistenza nei confronti dell'infanzia abbandonata e in seguito perse questa coloritura per assumere come obiettivi il controllo della salute e la tutela della razza. Questa istituzione nei confronti delle madri si poneva con intento di moralizzazione e di assistenza, senza prevedere finalità di emancipazione e di formazione; nei confronti dei/delle bambini/e era caratterizzata da finalità di igiene, di custodia, di assistenza senza alcuna finalità educativa. Una caratterizzazione purtroppo, che influì non poco sulla successiva storia delle istituzioni per la prima e la primissima infanzia in Italia.

L'O.N.M.I. venne fondata all'inizio per sostenere le famiglie bisognose che a causa delle condizioni di miseria materiale e sociale non erano in grado di accudire i propri figli, e quelli che in seguito sarebbero stati chiamati "casi sociali": in particolare si rivolgeva alle madri nubili, vedove, o con mariti che non provvedevano al mantenimento della famiglia (perché carcerati o invalidi...). In realtà, la sua diffusione fu capillare e Victoria De Grazia (1993) sottolinea come a causa di tale diffusione l'Opera ebbe un influsso consistente sui comportamenti e sulle convinzioni delle donne, anche perché intratteneva relazioni molto strette con medici, ostetriche, farmacisti e così via: diffondeva quindi nozioni di igiene, di 
puericultura, pratiche di cura prenatale, e post partum, in alcuni casi non prive di contraddizioni: si ricordi, ad esempio, il fatto che l'Opera promuoveva l'allattamento al seno ma in realtà distribuiva campioni gratuiti di latte in polvere Nestogen (Nestlé) incentivando l'utilizzo di questo prodotto.

Per quanto riguarda l'organizzazione e la gestione degli sili nido, l'O.N.M.I. nono operava nell'ottica del riconoscimento, del sostegno e della promozione dei diritti di bambine e bambine, di fatto vanificando e contraddicendo tutte le iniziative e i dibattiti fino a quel momento promossi dalle donne e dalle associazioni femminili: infatti non era rivolta a tutti/e ma soltanto a famiglie bisognose: l'offerta dunque era di un servizio di assistenza e privo di caratteri educativi. La dimensione pedagogica era del tutto oscurata da quella igienica, sanitaria e soprattutto di custodia: questo dato risulta evidente anche dall'organizzazione degli spazi e delle routines, molto centrati sui compiti del medico pediatra e non sui bisogni, interessi, desideri di bambini e bambine. Non erano previsti spazi per l'esplorazione autonoma da parte dei/delle piccoli/e, ma una struttura molto rigida a asettica, pensata per evitare contagi o incidenti e per controllare rigorosamente le azioni dei/delle piccoli/e ospiti. Susanna Mantovani (2006) sottolinea come questo fosse anche connesso alla preparazione del personale, che era molto sbilanciata sulle esigenze di sorveglianza e di igiene e che era prevalentemente infermieristica. Infine, è da rilevare la rigida separazione fra interno ed esterno: il nido era isolato dal resto del territorio e solo il personale aveva accesso.

Per tutti gli aspetti sopra elencati, l'O.N.M.I. costituiva un'istituzione del tutto differente dalle realizzazioni delle associazioni femminili e delle donne, e rappresentava un vistoso passo indietro riguardo al tema importantissimo del diritto all'educazione di bambini e bambine.

Curiosamente, l'O.N.M.I. che era nata con evidenti scopi stabiliti dal regime fascista, sopravvisse alla caduta del fascismo per molto tempo, fino al 1975, per complesse e molteplici ragioni e purtroppo per molti aspetti ebbe un'influenza molto pesante in Italia sul destino delle istituzioni per la prima infanzia.

I nuovi servizi, gli asili nido di tipo nuovo sorgeranno proprio cercando di differenziarsi da questa istituzione, ritenuta del tutto inadeguata per i bisogni e gli interessi di bambini e bambine: è interessante notare come anche la fondazione e la diffusione di questi ultimi sarà strettamente connessa, in Italia, con le iniziative, i dibattiti, le realizzazioni avanzate dalle donne e dalle loro associazioni.

\section{Referências}

CAGNOLATI, Antonella, COVATO, Carmela (a cura di), La scoperta del genere tra autobiografia e storie di vita. Sevilla: Editorial Benilde, 2015.

CAGNOLATI, Antonella, PINTO MINERVA, Franca, ULIVIERI, Simonetta (a cura di), Le frontiere del corpo. Mutamenti e metamorfosi. Pisa: Edizioni ETS, 2013. 
CAGNOLATI, Antonella (a cura di). Maternità militanti. Impegno sociale tra educazione ed emancipazione. Roma: Aracne Editrice, 2010.

CAMBI, Franco e ULIVIERI, Simonetta. Storia dell'infanzia nell'Italia liberale. Firenze: La Nuova Italia, 1988.

CATARSI, Enzo. L'asilo e la scuola dell'infanzia: storia della scuola "materna" e dei suoi programmi dall'Ottocento ai giorni nostri. Scandicci: La Nuova Italia, 1994.

CATARSI, Enzo. L'infanzia italiana dalla ruota all'asilo nido. In: BENIGNI, Laura (et al.). Un nido educativo. Milano: Fabbri, 1982.

CATARSI, Enzo, GENOVESI, Giuseppe. L'infanzia a scuola: l'educazione infantile in Italia dalle sale di custodia alla materna statale. Bergamo: Juvenilia, 1985.

CERISARA, Ana Beatriz. Professoras de educação infantil: entre o feminino e o profissional. São Paulo: Cortez, 2002.

CORSINI, Carlo. Infanzia e famiglia nel XIX secolo. In: BECCHI, Egle e JULIA, Dominique. Storia dell'infanzia. Roma-Bari: Laterza, 1996. pp. 250-281.

DE GIORGI, Fulvio. Maria Montessori modernista. Annali di storia dell'educazione e delle istituzioni scolastiche, Brescia: 2009, 16, 199-216.

DE GRAZIA, Victoria. Le donne nel regime fascista. Venezia: Marsilio, 1993.

DELLA PERUTA, Franco. Alle origini dell'assistenza alla prima infanzia in Italia. In: SALA LA GUARDIA, Lina e LUCCHINI Egidio (a cura di). Asili nido in Italia. Il bambino da zero a tre anni. Milano: Marzorati Editore, 1980. pp. 13-38

FILIPPINI, Nadia, PLEBANI, Tiziana (a cura di). La scoperta dell'infanzia: Cura, educazione e rappresentazione. Venezia: Marsilio, 1999.

FINCO, Daniela; SOUZA, Adalberto dos S., OLIVEIRA, Nara R.. Educação e Resistência escolar: gênero e diversidade na formação docente. Editora Alameda, 2017 (no prelo). 
GALLERANI, Manuela. Maria Montessori: “donna nuova” e intellettuale impegnata nella (ri)scoperta dell'infanzia. In: CAGNOLATI, Antonella. (a cura di). Maternità militanti: Impegno sociale tra educazione ed emancipazione. Roma: Aracne Editrice, 2010. pp. 83-114.

HONEGGER FRESCO, Grazia. Maria Montessori, una storia attuale. Roma-Napoli: Edizioni L'Ancora del Mediterraneo, 2008.

LOPEZ, Anna Grazia. Scienza, genere, educazione. Milano: Franco Angeli, 2015.

MACINAI, Emiliano. L'infanzia e i suoi diritti. Sentieri storici, scenari globali e emergenze educative. Pisa: ETS Edizioni, 2006.

MALNATI Linda. Il problema degli asili nido infantili. La difesa delle lavoratrici. Milano. 7 gennaio 1921, 2. MANTOVANI, Susanna. L'evoluzione del ruolo dell'educatrice nella storia del nido. In: TERZI, Nice (a cura di). Prospettive di qualità al nido. Il ruolo del coordinatore educativo. Bergamo: Edizioni Junior, 2006. pp. 47-64.

MERLO, Giordana. La prima infanzia e la sua educazione tra utopia e scienza dall'Età Moderna al Novecento. Milano: Franco Angeli, 2011.

MONTESSORI, Maria. L'autoeducazione. Milano: Garzanti: 1986.

MONTESSORI, Maria. La scoperta del bambino, Milano: Garzanti, 1980.

MUSI, Elisabetta. Non è sempre la stessa storia. Interrogare la tradizione, dar voce alle differenze di genere nelle pratiche educative. Milano: Franco Angeli, 2008.

PESCI, Furio. L'educazione morale e religiosa nell'opera di Maria Montessori, History of Education and Children's Literature. Macerata: dic. 2011, n. 2, 159-167.

PIRONI, Tiziana. Ellen Key e il femminismo italiano di inizio Novecento. In CAGNOLATI, Antonella (a cura di). Maternità militanti. Impegno sociale tra educazione ed emancipazione. Roma: Aracne Editrice, 2010. pp. 41-63.

PIRONI, Tiziana. Femminismo ed educazione in età giolittiana: Conflitti e sfide della modernità. Pisa: ETS Edizioni, 2010. 
PIRONI, Tiziana. Percorsi di pedagogia femminile. Dall'Unità d'Italia al secondo dopoguerra. Roma: Carocci, 2014.

REGNI, Raniero. Infanzia e società in Maria Montessori: Il bambino padre dell'uomo. Roma: Armando, 2007.

SEVESO, Gabriella. Insegnando. Adultità. Disagio e relazioni di aiuto, Milano. gennaio 2001, 13, 76- 87.

SEVESO, Gabriella e MAPELLI, Barbara. Una storia imprevista. Femminismi del Novecento ed educazione. Milano: Guerini Editore, 2003.

STEFANINI, Placida. Educhiamo la donna! La difesa delle lavoratrici. 6 aprile 1913, 1.

TRABALZINI, Paola. Maria Montessori. Da Il Metodo a La scoperta del bambino. Roma: Aracne, 2003.

ULIVIERI, Simonetta. (a cura di). Educazione al femminile. Una storia da scoprire. Milano, Guerini scientifica, 2007. 